\title{
Gender and Trauma in the Near-Death Experience: An Epidemiological and Theoretical Analysis
}

\author{
Linz Audain, J.D., Ph.D., M.D. \\ George Washington University, The Mandate \\ Corporation, Washington, DC
}

ABSTRACT: In this article, I explore the nature of the "fear-death experience" (FDE) by way of an epidemiological analysis, and discuss the FDE as one of several causal theories of the near-death experience (NDE). I then pursue two hypotheses: (1) if the FDE model is correct, one would expect to find that a number of NDEs are preceded by traumatic experiences; and (2) if the FDE model is correct, one would expect to find that more NDEs are experienced by males than females. Chi-squared analyses on data from more than 500 NDE cases revealed that the first hypothesis cannot be rejected, while the second hypothesis can be rejected. I discuss the theoretical implications of these findings.

One of the more interesting and recent hypotheses in the evolving scientific literature on the near-death experience (NDE) is the hypothesis that the experience may have little to do with objectively-defined imminent death. It appears that an NDE can be precipitated by the belief that one is dying. Ian Stevenson, Emily Cook, and Nicholas McClean-Rice (1989-90) have termed this the fear-death experience (FDE). The utility of identifying an FDE is that such an identification has the potential of directing the scientific community toward an accurate explanatory model of the NDE. This is possible because the analysis of an NDE as an FDE changes the analytical focus from imminent death to the perception of imminent death.

Linz Audain, J.D., Ph.D., M.D., is a Resident in Internal Medicine at George Washington University, and Chief Executive Officer of The Mandate Corporation. The author is grateful for comments he has received from Dr. Adnan Eldadah. Requests for reprints should be addressed to Dr. Audain at The Mandate Corporation, 4410 Massachusetts Avenue, Box 252, Washington, DC 20016. 
Accordingly, the purpose of this article is to pursue an understanding of the FDE by way of an epidemiological analysis. If the FDE hypothesis is correct, one would expect to find that the preponderance of NDEs had occurred incidental to trauma. That is, the individual who experiences sudden, life-threatening trauma is more likely to believe that death is imminent and is therefore more likely to fear it. Conversely, the individual who has a prolonged illness may have very little way of knowing when death is imminent and is therefore less likely to fear it. Under the FDE hypothesis, one would expect to find more NDEs among the former set of individuals.

A FDE hypothesis also suggests the possibility of a positive correlation between the frequency of NDEs and male gender. For social reasons one might expect males to be more likely to be involved in lifethreatening activities, such as war. Furthermore, one might also expect this to be true for biological reasons, such as increased testosterone level leading to more aggressive, life-threatening behavior.

Although it is easy to characterize the preceding propositions regarding the importance of gender and trauma to the incidence of the NDE, establishing their validity through a consultation of the NDE literature is a different matter altogether. A consultation of that literature reveals inconsistent conclusions among various investigators regarding the importance of gender and trauma to the incidence of the NDE. In many studies, there is no discussion at all of the importance of gender and trauma to the incidence of the NDE. Therefore, in the present article, I will attempt to reconcile these discordant strains in the neardeath literature by pooling the respective data from several studies and analyzing them in the spirit of statistical meta-analysis.

In view of the foregoing, I have organized this article as follows. In Part I, I will discuss explanatory models of the NDE and FDE. In Part II, I will present summaries of NDE studies that have presented data on gender and trauma. In Part III, I will pool the data from these and other studies and analyze the results. In Part IV, I will discuss these results.

\section{Explanatory Models of the NDE}

\section{Definition and Assessment of the NDE}

A threshold problem that is confronted by any study that attempts to pool NDE data is the problem of disuniform definitions and assessments of NDEs. With regard to definitions of the NDE, there are four such definitions that are relevant to this article. First, Raymond Moody's 
(1975) original characterization of the 15 elements of an NDE, such as a ringing noise and dark tunnel, laid a foundation for subsequent scientific work on NDEs. Yet even Moody was quick to point out that the NDEs of his interviewees were disuniform so that not all 15 elements were necessarily present in any given NDE. As a second definition, Kenneth Ring (1980) in his work with interviewees, recharacterized Moody's elements into the five stages of an NDE, such as euphoria and out-of-body experience.

A third definition of the NDE is that suggested by Michael Grosso (1981). Grosso maintained that "two types of NDEs" (p. 4) could be identified. The first type is the deathbed vision experienced at "the hour of death" by someone who is ill. The second type occurs where individuals suddenly find themselves in a life-threatening situation. This distinction between NDEs on the basis of their preconditions - that is, trauma versus nontrauma-is clearly the kind of distinction that is being pursued in this article.

A final definition of the NDE was suggested by Bruce Greyson (1985), who has proposed a typology of NDEs. According to this typology, there are three classes of experiences that are possible within the NDE. These types of experiences are transcendental, such as seeing mystical beings; affective, such as experiencing peace; and cognitive, such as reviewing one's life. What is significant for purposes of this article is that Greyson found that preconditions to the NDE affected the nature of the experiences that occurred during the NDE. Individuals who anticipated death, as in attempted suicide, were more likely to have transcendental and affective rather than cognitive experiences. Greyson also found that the subjects within each class of experience did not differ demographically within his sample of $\mathbf{8 9}$ cases across three classes of experiences.

With respect to the assessment of NDEs, there are two instruments that are currently used to achieve this goal. The first instrument, developed by Ring (1980), is his Weighted Core Experience Index (WCEI), which he developed from his interviews with NDEs. The second instrument, an NDE Scale developed by Greyson (1983) and tested on 67 subjects, is an instrument that has proven reliable in identifying the presence of an NDE. The WCEI remains useful for quantifying the depth of any given NDE.

As I will discuss further below, the pooling methodology of this article will involve a consideration of NDEs that occurred up to 250 years ago. Therefore, despite the meritorious nature of the WCEI and the NDE Scale, it will not be possible at this time to apply these instruments to 
the NDEs that pre-date them. For purposes of this article, I will assume that reported and recorded NDEs are valid NDEs.

\section{Traditional Neurobiological Models of the NDE}

It is useful to pause at this point to consider the broader background of explanatory models against which the present article is written. If one consults the NDE literature, it is possible to find a number of authors who argue that explanatory models of the NDE can be classified in one of two ways. For example, Susan Blackmore (1993) argued that there are "two alternative points of view" (p. 3) regarding the NDE. One point of view, the "afterlife hypothesis," maintains that the NDE is a real experience in which the "soul is travelling in a non-material world beyond the limitations of space and time" (p. 4). Another point of view, the "dying brain hypothesis," maintains that "[a]ll the phenomena of the NDE are believed to be products of the dying brain" (p. 4). Moody (1975) also argued that the choice between explanations of the NDE is a choice between "supernatural explanations" and "natural (scientific) explanations" (p. 156).

Unfortunately, one possibility that is ignored by this distinction between supernatural and natural explanations of the NDE, is the possibility that the NDE is a real supernatural event that cannot be explained within the confines of the present scientific technology. The history of science is littered with examples of scientific explanations that preceded the development of the appropriate technology (Audain, in press). For now, however, it is useful to briefly consider the nature of traditional explanations of the NDE whose purpose it is to explain the NDE without having to appeal to anything supernatural.

Moody (1975) presented a number of cogent arguments to refute the more traditional explanations of the NDE. For example, the pharmacological explanation of the NDE is untenable simply because many of his subjects had received no drugs when they experienced their NDE. Moreover, individuals who have taken hallucinogenic drugs have vague and varied experiences that do not resemble the NDE. The hypoxic brain explanation is untenable because many of the NDEs occurred before bodily injury or stress occurred to the body; that is, many of the NDEs were FDEs. "Autoscopic hallucinations" are distinguishable from the NDE on phenomenological grounds; for example, in the autoscopic hallucination, the hallucinating individual sees him- or herself alive, from the neck up, conversing; there are no associated features such as a life review, tunnel, or beings of light. 
Parenthetically, in addition to the traditional explanation of the NDE, there are purely psychological explanations of the NDE that Moody (1975) also refuted. I will not discuss these explanations here, for in the final analysis, they also must be premised on some underlying biological or physical explanation of the NDE.

The practice of proposing biological or physical explanations of the NDE without appealing to the supernatural is a practice that continues unabated. This is as it should be, for it is unlikely that there will be a scientific understanding of the NDE without such a pursuit of knowledge and truth. Having said that, it is equally clear that there are two phenomena within the NDE that cannot be explained by traditional explanations. These are the veridical observation phenomenon and the precognition phenomenon. I will consider each of these phenomena briefly below, followed by a discussion of three of the more recent traditional explanations of the NDE; and I will then discuss yet a third phenomenon: the fear-death experience phenomenon.

\section{The Veridicality and Precognition Phenomena}

The veridicality phenomenon arises when NDErs recount things that they observed during their NDE, and these observations are subsequently confirmed. However, under present scientific theories, it is not possible to explain how these observations could have been made while the individual was without vital signs and being resuscitated. Moody (1988) recounted four such experiences. For example, in one experience, the subject not only described all of the resuscitation procedures that were performed on him, but he also described the emergency room nurse and gave her name; while out of his body he had walked through her in the hallway and had read her name tag. In another experience reported by Moody, a woman who had been blind for 50 years was able to describe the resuscitation procedures, equipment, and other items in the room during her NDE, things that she could not possibly have known anything about because of her lifelong blindness. The NDE literature continues to report these veridical observations (Ring and Cooper, 1997; Ring and Lawrence, 1993).

The precognition phenomenon arises when the experiencer returns from the NDE with predictions about the future, and these predictions come true without the intervention of the subject. Perhaps the most celebrated case of this is that of Dannion Brinkley, one of Moody's subjects, who apparently predicted the downfall of the Soviet Union and the Persian Gulf War 14 and 15 years before their occurrence 
(Brinkley and Perry, 1994). Ring (1982) has characterized these precognitions as being either "personal flashforwards" if they pertain to the subject's personal life or "prophetic visions" if they are predictions on a societal or planetary scale. Craig Lundahl (1993) has recently identified yet a third kind of precognition: an "otherworld personal future revelation," similar to flashforwards but different in that they are received while the subject is in the "otherworld" and not during the life review.

Explanations of the veridicality and precognition phenomena continue to elude traditional explanations of the NDE. For example, Melvin Morse, David Venecia, and Jerrold Milstein (1989) have proposed that NDE-like memories are genetically encoded in the temporal lobe and are activated by serotonergic mechanisms that in turn are activated by physiological stress. Although they discuss hallucinations, nowhere in the article do they discuss the veridicality or precognition phenomena. Indeed, how could parents pass on genes that contain specific knowledge of medical technology, years before the invention of the equipment, permitting, for example, veridical observation of resuscitation equipment?

Similarly, Jean-Pierre Jourdan (1994) has argued that much of the $\mathrm{NDE}$ can be explained through an analysis of the $\mathrm{N}$-methyl-D-aspartate (NMDA) receptors that are found in the hippocampus. It has been established that these receptors are important in the long-term potentiation of hippocampal neurons, resulting in the formation of memories. Jourdan theorized that the "sensory disconnection" of an NDE causes the release of "neuroprotective substances" that block the NMDA receptors, thereby precluding long-term potentiation. As a consequence, "internal memories...take the place of the missing sensory input" (Jourdan, 1994, p. 191). Unfortunately, when considered in the light of the veridicality phenomenon, Jourdan's theory suffers from the same temporal difficulty as Morse's theory: how can the subject's brain have a precise internal memory of things that were never neuronally encoded and had no way of being encoded?

Finally, one of the more interesting, recent, and direct efforts to explain the veridicality phenomenon is found in the work of Juan GómezJeria and Juan Saavedra-Aguilar (1994). They argued that recall of NDE events involves the "priming" of memory through the use of conscious and unconscious environmental stimuli. The individual is particularly attuned to these stimuli during the altered NDE state. This information is combined with previous knowledge to give a "story" that is highly credible. There is enormous difficulty here: how do these 
authors explain to Ring and Madelaine Lawrence's (1993) patient the nature of the stimuli in the emergency room that allowed her to see the red shoe in the corner of the hospital roof while she was out of her body?

\section{"Fear-Death" Models of the NDE}

The fear-death experience represents one of the more interesting and recent challenges to the NDE scientific community. In a seminal study, Stevenson, Cook, and McClean-Rice (1989-90) examined the medical records of NDErs to discover whether those subjects were medically and objectively near death. They discovered that 45 percent of the patients were objectively near death, while 55 percent were not. However, 83 percent of the patients believed they had been near death. These data led Stevenson and his colleagues to conclude that not all NDErs are necessarily near death. They concluded that those experiences that occur when the subject is not near death might be more appropriately labeled the "fear-death experience."

More recently, Glen Gabbard and Stuart Twemlow (1991) reviewed the literature on the FDE, concluding that the "key determinant" of the NDE is the "perception of being near death, independent of the actual reality of the situation" (p. 41). In discussing the FDE, Gabbard and Twemlow cited their earlier study of 339 subjects with out-of-body experiences (Twemlow, Gabbard, and Jones, 1982). From that study, they concluded that none of the characteristics of the NDE was "exclusive" to the NDE, although many of them were "significantly more likely to occur" when the subject believed death was imminent (Gabbard and Twemlow, 1991, p. 42). Unfortunately, they were led to conclude that the NDE or out-of-body experience is a psychological defense mechanism that protects the individual from imminent death. Neither the veridicality nor the precognition phenomenon was discussed within their review. Further, Gabbard and Twemlow were forced to speculate mightily as to how a $2 \frac{1}{2}$-year-old child who had an NDE while having no conception of death was able to develop such an elaborate psychological defense mechanism.

Even more recently, Keith Floyd (1996) demonstrated that precognition is possible within an FDE. In the case reported by Floyd, a patient who was scheduled to receive electroconvulsive therapy for her depressive symptoms was opposed to the therapy and believed she was going to die upon receiving it. Shortly after receiving the electric shock, the patient had an NDE with a precognition that was confirmed two years later. Floyd concluded that one possible explanation for the NDE was 
the patient's belief that she was going to die once she received the electric shock.

In conclusion, the intellectual challenge that faces the scientific community is to develop theoretical models that explain the FDE while simultaneously addressing the veridicality and precognition phenomena. These models can perhaps best be appreciated against a background of empirical data. It is to a discussion of these data that I now turn.

\section{Epidemiological Studies of the NDE}

\section{The Moody Data (1975)}

There can be little doubt that Moody's 1975 publication of his interviews of more than 150 NDErs represents one of the seminal works in the development of the NDE literature. Unfortunately, Moody's book offered no tabulation of his reported cases according to gender or the presence of trauma as the precondition to the NDE. Moody did, however, offer an opinion, based on his experience, that NDEs were underreported by males. Notwithstanding this epidemiological limitation of Moody's book, it is possible to analyze the cases he discussed to draw some conclusions as to the gender of the subject and the existence of trauma or nontrauma as the precondition to the NDE. To be sure, this analysis was not possible in each of Moody's reported cases. However, of 59 cases in which gender could be identified, 31 of the subjects were female while 28 were male. Of 25 cases in which NDE preconditions could be identified, 22 NDEs were precipitated by trauma, while 3 were precipitated by nontraumatic circumstances.

\section{The Ring Data (1980)}

Ring's 1980 analysis of 104 NDE cases from 102 subjects is thought to be the "first major study to quantify and analyze NDE data" (Basford, 1990 , p. 59). Moreover, in his study, Ring considered the specific questions regarding gender and trauma that are the focus of the present article. Of his 102 subjects, 57 were female, while 45 were male. With regard to the preconditions to the NDE, 52 subjects had illness as a precondition, while 26 subjects experienced an accident and 24 attempted suicide. Unfortunately, nowhere in his book did Ring define what constituted an "illness." It is difficult to know therefore how many of Ring's illness- related NDEs might have been precipitated by trauma. 
Notwithstanding this limitation, it is significant to note that Ring detected some gender differences in his study. Specifically, he found that the NDEs of women were more often associated with illness while those of men were more often associated with accident and suicide.

\section{The Sabom Data (1982)}

Sabom's (1982) investigation of 116 subjects has been called "the most rigorous medical inquiry [of NDEs] to date" (Basford, 1990, p. 80). Of the 33 subjects who experienced NDEs, 26 subjects experienced NDEs after cardiac arrest, 2 experienced NDEs after accidents, while 5 experienced NDEs in a comatose state. In terms of gender distribution, 26 of the patients were male, while 7 were female.

\section{The Twemlow and Gabbard Data (1984)}

Twemlow and Gabbard (1984) reported the results of a survey that had been sent to 700 individuals who had claimed to have out-of-body experiences. Of the 700, 34 had had NDEs. The objective of Twemlow and Gabbard's study was to determine the effect of demographic and preexisting conditions on the NDE. Of the 34 NDErs, there were 22 women and 12 men. Regarding preexisting conditions, 9 of the subjects had experienced illnesses prior to the NDE, while 25 had experienced physical trauma of some kind, such as eclamptic convulsion or surgery. Twemlow and Gabbard's results regarding gender differences in preexisting conditions were consistent with Ring's (1980): their male subjects were more likely to have had their NDEs as a result of accident or physical injury.

\section{Other Data}

Two other broad sources of data are worth mentioning at this point. The Gallup Poll (Gallup and Proctor, 1982) surveyed 1,500 adult Americans and concluded that approximately 5 percent of the American population had experienced an NDE. They reported that the incidence of NDEs was approximately equally distributed across genders. However, NDEs were more likely to be induced by trauma, such as accidents or childbirth, than by close brushes with death without trauma. The Gallup Poll has been called "[t]he most comprehensive survey of NDE incidence to date" (Basford, 1990, p. 75). 
The other source of data is the bibliography of the NDE literature by Terry Basford (1990), a comprehensive annotated bibliography of the NDE literature from 1847 to 1989 . A count of the published NDEs summarized by Basford for which gender data were available reveals that 43 of the subjects experiencing NDEs were men, while 12 were women. With regard to preconditions, a count of cases in which precondition data were available reveals that $35 \mathrm{NDEs}$ were induced by trauma, while 13 were induced by nontraumatic circumstances.

\section{Gender and Trauma in the NDE: An Epidemiological Analysis}

\section{Method}

The basic approach pursued in this study was to pool data, from as many sources as possible, related to the gender distribution and incidence of trauma associated with NDEs. The data presented above are the major studies relied upon for purposes of this article. I retrieved additional data from (1) studies listed in the Psychological Abstracts that were accessible; (2) studies listed in the Basford (1990) bibliography that were accessible; (3) all studies published in the Journal of Near Death Studies from 1988 to 1996; and (4) studies published in the bibliography of the recent NDE anthology compiled by Lee Bailey and Jenny Yates (1996).

The underlying analytical methodology that informed the approach taken in this article was the methodology of meta-analysis, defined as "the analysis of analyses." The basic method of meta-analysis is that "[e]ach data point used for analysis is obtained from an individual study rather than from an individual subject" (Wolf, 1986, p. 11).

Strictly speaking, the pooling of raw data that I pursued in the present paper is not meta-analysis as it is currently practiced, which involves the analysis of test statistics from individual studies (Petitti, 1994, pp. 15-19). The reality, however, is that the field of near death studies is very much in its scientific infancy, and in many of the studies discussed above and in those to be discussed below, test statistics and control samples are absent. In light of this, the solution I pursued in this article is a second-best solution in the form of an analysis of the raw data from individual studies. It is clear that there are potential problems with this pooling methodology. For example, whatever selection bias may have been present in the original raw data is perpetuated in 
Table 1

Gender and Trauma in the NDE

\begin{tabular}{lrrrrrc}
\hline & \multicolumn{2}{c}{ Gender } & & \multicolumn{2}{c}{ Precondition } \\
\cline { 2 - 3 } \cline { 5 - 6 } Study & Male & Female & & Traumatic & Nontraumatic \\
\hline Moody (1975) & 28 & 31 & & 22 & 3 \\
Ring (1980) & 45 & 57 & & 52 & 50 \\
Sabom (1982) & 26 & 7 & & 5 & 28 \\
McLaughlin and Maloney (1984) & 12 & 28 & & \\
Twemlow and Gabbard (1984) & 12 & 22 & & 25 & 9 \\
Basford (1990) & 43 & 12 & & 35 & 13 \\
Morse and Perry (1990) & 6 & 4 & & 6 & 2 \\
Serdahely (1990) & 1 & 2 & & 3 & 0 \\
Sutherland (1990) & 15 & 35 & & \\
Serdahely (1991) & 5 & 5 & & \\
Gibson (1994) & 28 & 40 & & \\
Pacciolla (1996) & 38 & 26 & & \\
\hline
\end{tabular}

the meta-analysis. Yet because of the paucity of data on NDEs, it would seem better to have some possibly biased data, rather than no data at all. Moreover, it is possible that some of the biases of the individual authors will cancel out one another.

\section{Data and Results}

The data on gender and the presence of a trauma precondition in NDEs are presented in Table 1.

With respect to gender, the total number of males from Table 1 is 259 , while the total number of females is 269 . This gender difference was not statistically significant (chi-squared $=0.19, \mathrm{df}=1$ ). Therefore, we cannot reject the null hypothesis that there is no difference between the number of males and females who experience the NDE; the present data suggest but does not prove that the numbers of males and females who have NDEs are comparable. This result is consistent with the majority of the studies discussed above.

With respect to the incidence of trauma as a precondition to an NDE, the total in the traumatic precondition column is 148 and the total in the nontraumatic column is 105 . This difference in preconditions was statistically significant (chi-squared $=7.68, \mathrm{df}=1, \mathrm{p}<.01$, one-tailed). 
Therefore, the null hypothesis that there is no difference between trauma and nontrauma as a precondition for an NDE can be rejected; the present data suggest that the incidence of NDEs is higher in the presence of trauma than in its absence. It is important to note that the chi-squared analysis cannot be used to support a claim about the relative importance of trauma to the incidence of NDEs, because the total number of traumatic and nontraumatic brushes with death is not known.

\section{Discussion}

What the preceding data make clear is that a pressing challenge to any model of the NDE is to explain why trauma might be important to the incidence of NDEs. The discussion above suggests a first approximation to an answer: namely, that if the near-death experience is in reality a fear-death experience, then it follows that unexpected, lifethreatening, traumatic situations, in which one would be inclined to fear death the most, would be expected to precipitate more NDEs than would nontraumatic circumstances. The data suggest that this will be true irrespective of the gender of the individual.

In view of this first approximation to understanding the importance of trauma to the incidence of NDEs, as a second approximation, the question that now arises is this: How is the human organism physiologically different in a condition in which it is fearing death? There are at least three possible answers to this question, any combination of which could conceivably be correct. Specifically, the fear of death might bring forward within the organism (1) endogenous opioids, (2) limbic involvement, and/or ( 3 ) adrenergic involvement.

Other investigators have commented on the possible role of endogenous opioids and the limbic system in triggering a NDE (SaavedraAguilar and Gómez-Jeria, 1989). However, in view of the data in this article and the FDE data, what one must postulate is that there is release of opioids and limbic involvement in anticipation of the pain that the traumatic experience will inflict upon the organism, such as during the fall from the cliff, or before a grenade explodes. A similar physiological story can be told about the release of adrenergic neurotransmitters: the release of epinephrine and norepinephrine from the adrenal medulla can occur rather instantaneously in anticipation of a fight or flight response. Two decades before these experiences were named NDEs, Carl Jung (1955) speculated on a possible role for the 
sympathetic system in bringing on such experiences. Unfortunately, other investigators have not pursued that line of reasoning.

If the foregoing considerations are correct, then if individuals are able voluntarily to control the release of these chemical substances in the right mix and quantities, then they could achieve voluntary NDEs. This would explain why the individual who has been trained to achieve an out-of-body experience can have an experience that is similar to an NDE.

Yet the explanatory story regarding the NDE cannot end here. It will be recalled from the discussion above that the pressing challenge faced by an explanatory model of the NDE is to address the veridicality, precognition, and fear-death phenomena. An explanatory model that is based only on neurotransmitters is forced to ignore or dismiss the veridicality and precognition phenomena because it is unable to explain them. In searching for more robust explanatory models, one possibility is to look for explanations that integrate, but do not rely solely on, neurotransmitters, such as explanations beyond the third dimension. A discussion of such an explanation is beyond the scope of the present article. However, elsewhere I have discussed some of the possibilities for such an explanation (Audain, in press).

\section{Conclusion}

The purpose of this article has been to address the role that gender and a precondition of trauma play in the incidence of NDEs. Having empirically addressed the nature of that role, I attempted to explain from a theoretical standpoint why trauma might be a precondition to the onset of an NDE. It seems to me relatively clear that the debate over the etiology of the NDE is a debate that will continue for some time. I can only hope that this article has made a small contribution toward the clarification of the issues within that debate.

\section{References}

Audain, L. (In press). Near-death experiences and a theory of the extraneuronal hyperspace. Journal of Near-Death Studies.

Bailey, L. W., and Yates, J. (1996). The near-death experience: A reader. New York, NY: Routledge.

Basford, T. K. (1990). Near-death experiences: An annotated bibliography. New York, NY: Garland. 
Blackmore, S. J. (1993). Dying to live: Near-death experiences. Buffalo, NY: Prometheus.

Brinkley, D., and Perry, P. (1994). Saved by the light: The true story of a man who died twice and the profound revelations he received. New York, NY: Villard.

Floyd, K. (1996). ECT: TNT or TLC? A near-death experience triggered by electroconvulsive therapy. Journal of Near-Death Studies, 14, 187-195.

Gabbard, G. O., and Twemlow, S. W. (1991). Do "near-death experiences" occur only near death?-Revisited. Journal of Near-Death Studies, 10, 41-47.

Gallup, G., and Proctor, W. (1982). Adventures in immortality: A look beyond the threshold of death. New York, NY: McGraw-Hill.

Gibson, A. S. (1994). Near-death experience patterns from research in the Salt Lake City region. Journal of Near-Death Studies, 13, 115-127.

Gómez-Jeria, J. S., and Saavedra-Aguilar, J. C. (1994). A neurobiological model for neardeath experiences. II: The problem of recall of real events. Journal of Near-Death Studies, $13,81-89$.

Greyson, B. (1983). The Near-Death Experience Scale: Construction, reliability, and validity. Journal of Nervous and Mental Disease, 171, 369-375.

Greyson, B. (1985). A typology of near-death experiences. American Journal of Psychiatry, $142,967-969$.

Grosso, M. (1981). Toward an explanation of near-death phenomena. Anabiosis: The Journal of Near-Death Studies, 1, 3-26.

Jung, C. G. (1955). The interpretation and nature of the psyche. New York, NY: Pantheon.

Lundahl, C. R. (1993). Otherworld personal future revelations in near-death experiences. Journal of Near-Death Studies, 11, 171-179.

McLaughlin, S. A., and Maloney, H. N. (1984). Near-death experiences and religion: A further investigation. Journal of Religion and Health, 23, 149-159.

Moody, R. A. (1975). Life after life. Covington, GA: Mockingbird Books.

Moody, R. A., and Perry, P. (1988). The light beyond. New York, NY: Bantam.

Morse, M. L., and Perry, P. (1990). Closer to the light: Learning from the near-death experiences of children. New York, NY: Villard.

Morse, M. L., Venecia, D., and Milstein, J. (1989). Near-death experiences: A neurophysiologic explanatory model. Journal of Near-Death Experiences, 8, 45-53.

Pacciolla, A. (1996). The near-death experience: A study of its validity. Journal of NearDeath Studies, 14, 179-185.

Petitti, D. B. (1994). Meta-analysis, decision analysis, and cost-effectiveness analysis. New York, NY: Oxford University Press.

Ring, K. (1980). Life at death: A scientific investigation of the near-death experience. New York, NY: Coward, McCann and Geoghegan.

Ring, K. (1982). Precognitive and prophetic visions in near-death experiences. Anabiosis: The Journal of Near-Death Studies, 2, 47-74.

Ring, K., and Cooper, S. (1997). Near-death and out-of-body experiences in the blind: A study of apparent eyeless vision. Journal of Near-Death Studies, 16, 101-147.

Ring, K. and Lawrence, M. (1993). Further evidence for veridical perceptions during near-death experiences. Journal of Near-Death Experiences, 11, 223-229.

Saavedra-Aguilar, J. C., and Gómez-Jeria, J. S. (1989). A neurobiological model for neardeath experiences. Journal of Near-Death Studies, 7, 205-222.

Sabom, M. B. (1982). Recollections of death: A medical investigation. New York, NY: Harper and Row.

Serdahely, W. J. (1990). Pediatric near-death experiences. Journal of Near-Death Studies, 9, 33-39.

Serdahely, W. J. (1991). A comparison of retrospective accounts of childhood near-death experiences with contemporary pediatric near-death experience accounts. Journal of Near-Death Experiences, 9, 219-224.

Stevenson, I., Cook, E. W., and McClean-Rice, N. (1989-90). Are persons reporting "neardeath experiences" really near death? A study of medical records. Omega 21, 45-54. 
Sutherland, C. (1990). Changes in religious beliefs, attitudes, and practices following near-death experiences: An Australian study. Journal of Near-Death Studies, 9, 21-31. Twemlow, S. W., Gabbard, G. O., and Jones, F. C. (1982). The out-of-body experience: A phenomenological typology based on questionnaire responses. American Journal of Psychiatry, 139, 450-455.

Twemlow, S. W., and Gabbard, G. O. (1984). The influence of demographic/psychological factors and preexisting conditions on the near-death experience. Omega, 15, 223-235. Wolf, F. M. (1986). Meta-analysis: Quantitative methods for research synthesis. Beverly Hills, CA: Sage Publications. 